\title{
Getting Back on Their Feet: Women in Sport and Exercise Academic Network Virtual Conference, Sept 7-8, 2020
}

\author{
Jacky Forsyth \\ Staffordshire University \\ Rachael Bullingham \\ University of Gloucestershire
}

\author{
Nicola Brown \\ St. Mary's University \\ Claire-Marie Roberts \\ The Premier League
}

\section{Background to the Network and Conference}

The overall purpose of the Women in Sport and Exercise Academic Network (WiSEAN) is to grow, strengthen, and promote research on women in sport and exercise, with the ultimate goal of optimizing women's athletic success and their sport participation. The catalyst for the development of the network was the underrepresentation of female participants in sport and exercise medicine research (Costello, Bieuzen, \& Bleakley, 2014). Due to this underrepresentation, research evidence based on men is often inappropriately generalized and applied to women (Sims \& Heather, 2018). Gender bias is also apparent in conference speakers and academic panel members (Bekker et al., 2018) as well as in leadership positions in sport (Pape, 2020), where males consistently outnumber females. The network, therefore, exists to help minimize gender inequalities in research and academia, through promoting female-focused research as part of the annual conference and by supporting female researchers through its mentoring scheme. Further activities of WiSEAN can be found on the website: https://www.wisean.net/.

\section{Introduction to the 2020 Conference: Getting Back on Their Feet}

Owing to COVID-19, which in many countries led to restrictions regarding travel and public gatherings, the planned 2020 face-to-face conference was replaced with a free virtual event, hosted by Staffordshire University, UK. With early research evidence suggesting that COVID-19 had heightened gender inequalities in sport, employment, and academia (Bowes, Lomax, \& Piasecki, 2020; Collins, Landivar, Ruppanner, \& Scarborough, 2020; Oleschuk, 2020), the focus of the conference, 'getting back on their feet', aimed to virtually connect individuals from a range of disciplines to ensure that sport and exercise research had a focus on women during COVID-19 and beyond. The conference also provided networking opportunities to connect with others in sport and

All authors are with the Women in Sport and Exercise Academic Network. Forsyth is with Staffordshire University, Stoke-on-Trent, United Kingdom. Brown is with St. Mary's University, Twickenham, United Kingdom. Bullingham is with the University of Gloucestershire, Gloucestershire, United Kingdom. Roberts is with The Premier League, United Kingdom. Forsyth (j.j.forsyth@staffs.ac.uk) is corresponding author. exercise, as well as providing training and motivation through knowledge gained via others' research and experience. The Conference Scientific Committee comprised Dr. Jacky Forsyth (Staffordshire University), Dr. Rachael Bullingham (University of Gloucestershire), Dr. Nicola Brown (St Mary's University), and Dr. Claire-Marie Roberts (Premier League, UK), with technical direction from Mr. Matthew CoombeBoxall (Staffordshire University).

\section{Conference Review}

A total of 474 delegates registered for the event from 37 different countries. Most delegates were from the United Kingdom, with high numbers also joining from the United States, Qatar, and Australia. The virtual conference was specifically designed to allow delegates the flexibility to join sessions ad hoc, with sessions running over an extended time period to accommodate international time zones. Consequently, the number of delegates viewing each session varied throughout the course of the conference, reaching a peak of 126. Given this was WiSEAN's first online event, the Conference Committee were keen to obtain feedback from delegates to feedforward for future virtual events. Conference evaluations were overwhelmingly positive, with the breadth, quality, and style of presentations rated highly. All those who completed the survey said that they would recommend the event to others. Some examples of the comments left by delegates are as follows:

Brilliant online conference with a great variety of speakers and topics. I'll be taking away lots of learning and potential contacts to follow up with!

The ability to have some of the world's leading experts because of the virtual nature of the event was a strength of the conference.

Having people from all different backgrounds/disciplines but all working towards increasing the presence of women in research was inspiring.

I found the conference inspirational and stimulating and has fired me up to be an advocate for more research on females and ensure research on females is better represented in our curriculum.

Suggested areas for improvement included more opportunities for collaborative and face-to-face (online) communication via the chat facility, 
which was difficult to negotiate for some delegates. Additionally, comments were received about the duration of the conference days, lasting all day and into the evening; however, in order to accommodate international speakers and delegates, this was unavoidable.

\section{Looking Ahead}

The fourth WiSEAN conference will take place on April 19-22, 2021 and will be hosted virtually by the University of Worcester, UK. This conference will maintain the multidisciplinary approach of previous years and will cover all aspects of women's involvement in sport. The conference will have an international focus and aim to bring together practitioners and academics from a range of disciplines to allow discussion and debate surrounding women's participation in sport, exercise, and physical activity. The conference has been titled 'Redressing the Balance' and the purpose of the conference is to raise awareness and address inequalities from both an academic's and practitioner's perspective, in addition to assessing impact and sharing areas of good practice. On day four, there will be a parallel virtual conference, as part of a European Commission-funded Erasmus+ project which the University of Worcester is leading with six other international partners. The project is entitled the 'Gender Equity Toolkit for Generation Z' (an educational online resource via a MOOC [massive open online course]) and aims to educate Generation $\mathrm{Z}$ in issues of equity in sport with the purpose of increasing the number of women and girls in all forms of sport leadership and management. The impact and implications for practice from this three-year project will be shared along with key research studies focusing on gender perceptions, communication, and Generation Z.

\section{References}

Bekker, S., Ahmed, O.H., Bakare, U., Blake, T.A., Brooks, A.M., Davenport, T.E., . . . Whittaker, J.L. (2018). We need to talk about manels: The problem of implicit gender bias in sport and exercise medicine. British Journal of Sports Medicine, 52(20), 1-2. doi:10.1136/ bjsports-2018-099084

Bowes, A., Lomax, L., \& Piasecki, J. (2020). The impact of the COVID-19 lockdown on elite sportswomen. Managing Sport and Leisure. Advance online publication. doi:10.1080/23750472.2020.1825988

Collins, C., Landivar, L.C., Ruppanner, L., \& Scarborough, W.J. (2020). COVID-19 and the gender gap in work hours. Gender, Work \& Organization. Advance online publication. doi:10.1111/gwao.12506

Costello, J.T., Bieuzen, F., \& Bleakley, C.M. (2014). Where are all the female participants in sports and exercise medicine research? European Journal of Sport Science, 14(8), 847-851. doi:10.1080/ 17461391.2014.911354

Oleschuk, M. (2020). Gender equity considerations for tenure and promotion during COVID-19. Canadian Review of Sociology, 57(3), 502515. doi:10.1111/cars.12295

Pape, M. (2020). Gender segregation and trajectories of organizational change: The underrepresentation of women in sports leadership. Gender \& Society, 34(1), 81-105. doi:10.1177/0891243219867914

Sims, S.T., \& Heather, A.K. (2018). Myths and methodologies: Reducing scientific design ambiguity in studies comparing sexes and/or menstrual cycle phases. Experimental Physiology, 103(10), 1309-1317. doi:10.1113/EP086797 


\section{Keynote Abstracts}

A number of keynotes were presented over the two-day 2020 WiSEAN conference with academics, practitioners, and athletes from across the globe sharing their research, insights, and experiences. Summaries of the keynotes are presented below.

Becoming a Mother Doesn't Stop You Being an Athlete: My Journey Through Ultrarunning, Motherhood and Campaigning for Change

Sophie Power ${ }^{1}$

${ }^{1}$ Women in Sport

In this presentation, Sophie Power shared her journey as an athlete - from being "unsporty" as a child to completing some of the hardest ultramarathons in the world. An insight was given into the world of ultrarunning and the physical and mental demands of nonstop racing over multiple days. Sophie also spoke about how she trained for and completed the Ultra-Trail du Mont-Blanc, a 106-mile mountain race, just three months postpartum. The iconic photograph of her breastfeeding during the race, and the global discussion it launched, have seen Sophie become an advocate for mothers as athletes. As a trustee of Women in Sport, Sophie also touched on the importance of female-led research, which will benefit women from grassroots to elite, at all stages of life.

How Do We Get SmartHER in Our Support of Female Athlete Health, Wellbeing and Performance?

Dr Emma Ross ${ }^{1}$

${ }^{1}$ Thrive Revolution

At the start of the Tokyo Olympiad, in 2017, the UK High Performance System developed a campaign which aimed to optimize the support of elite female athletes. For a long time in sport, women have been treated and trained in ways that have been successful for their male counterparts, but which might not be optimal for a female athlete. Dr Ross, who developed and led the SmartHER campaign, talked about what insight informed the SmartHER work and how the campaign challenged the status quo within elite sport by asking coaches, athletes and practitioners to consider the 'female' as well as the 'athlete' of the individuals they supported. The SmartHER campaign is so-called because the aim was for everyone to be smarter about female-specific factors such as the menstrual cycle, breast support and pelvic health, and to consider how that new understanding is used in supporting athletes, whether as a coach or performance support practitioner. This presentation explored the opportunities and challenges that were encountered on the SmartHER journey. The importance of elite sport as a vehicle for change right across the participation to performance pathway, by inspiring and empowering girls and women to develop lifelong relationships with sport and exercise, was also discussed.

Researching Women in Sport and Exercise: Lessons Learnt From East Yorkshire to the Middle East

Dr Bryna Chrismas ${ }^{1}$

${ }^{1}$ Qatar University

Women and girls are typically less active than men and boys across the globe. And, there are unique barriers and challenges for girls and women, which may be further exacerbated across the Middle East. Elite women's sport is on the rise, and with global events taking place in hot and humid countries, it is essential to understand how to prepare female athletes for these conditions and to identify specific strategies for elite females to enhance their performance. As an exercise physiologist with experience of academia in the UK and Qatar, Dr Chrismas discussed her research on women in sport and exercise across health and performance. She also drew upon key events, research and initiatives that could be used to address the gender inequalities, and suggested a multi-disciplinary, holistic and collective approach to research in women across sport and exercise.

Optimizing Dancer Health and Performance in the Time of COVID-19 Dr Nicky Keay ${ }^{1}$

${ }^{I}$ Durham University, UK

The demands of dance are increasing over the generations. Yet, dance is often seen as the poor relation of sport. Female dancers, like female athletes, can struggle to find evidence-based strategies to optimize health and performance, as scientific and medical research is more focused on men. Dance requires a unique combination of athleticism and artistry. Due to the aesthetic requirement of dance, dancers are at risk of relative energy deficiency in sport (RED-S). In this presentation, Dr Keay discussed evidence for the consequences of low energy availability from previous studies in both young pre-professional and retired dancers. The latest results from a study assessing risk of RED-S in the international dance community were presented for the first time, revealing the psychological drivers. The results of this study are particularly relevant in the time COVID-19 where professional dancers are only just returning to the studio, whilst theatres remain shut indefinitely. Comparison was made with extensive guidelines for return to training and restarting of competition in sport.

\section{The Gendered Politics of Recovery: A Feminist Analysis of Women's Sport and Exercise Through COVID-19}

Professor Holly Thorpe ${ }^{1}$

${ }^{I}$ Te Huataki School of Health at the University of Waikato, New Zealand

In this talk, Professor Thorpe brought an interdisciplinary feminist perspective to understand the challenges for women in navigating time, space and resources for sport and exercise at various stages during the COVID-19 recovery response. Drawing upon two separate projects (an international survey with $>1000$ elite and recreational sportswomen, and interviews with women athletes and those working in the New Zealand sport and exercise industry), she revealed how women's opportunities, challenges and strategies for maintaining their sport and exercise participation were significantly impacted by economic (i.e., family incomes, job losses, limited budgets) and emotional (i.e., coping strategies, support structures) resources. Despite such challenges, many women took to developing a range of highly creative strategies to navigate time and space for ongoing participation. Both projects reveal the significant impact of the pandemic on women's training practices, everyday work and family routines, body image and modes of communication, as well as the emergence of new 'ethics of care' to support themselves and others during times of extreme personal and professional difficulty. Focusing on the impact of COVID-19 on the everyday sporting lives and careers of women, Professor Thorpe offered insights that can inform the recovery process, and towards more equitable policies and processes supporting women in the sport and exercise sector during the challenging times ahead.

\section{Why We Need to Undertake More Research on Female Athletes}

Professor Louise Burke ${ }^{1}$

\section{${ }^{1}$ Australian Catholic University, Australia}

Females are under-represented in sports science research, with few studies solely involving female participants and even fewer being organized to investigate differences in the response between male and female athletes. The theme of greatest inequality involves interventions for performance enhancement. Professor Burke reflected on her personal experience to identify a number of explanations for the relative scarcity of female 
participants in sports science research; these included greater difficulty in recruiting females, smaller numbers of females of elite caliber, the need to account for differences in menstrual status or stage of menstrual cycle, and difficulty in finding characteristics to allow matching with male athletes. Yet, it is important to undertake studies with female athletes if sports nutrition guidelines are to be realistically applied to this population. There are several reasons to consider that females may have special or different needs; these include differences in body size, the effects of specific sex hormones in the particular phase of the menstrual cycle, differences in nutritional status (e.g., energy availability or iron status) or other factors yet to be identified. Newer research on some sports nutrition practices - for example, the effectiveness of caffeine supplementation on endurance sports - has shown similar responses in male and female participants. Yet, in other areas - for example, nitrate metabolism and response to nitrate supplementation - there is preliminary evidence of differences between males and females. This presentation represented a call to arms for more research on female athletes.

\section{The Effects of Female Hormones on Responses to Resistance Training}

Dr Xanne Janse de Jonge ${ }^{1}$ and Dr Belinda Thompson ${ }^{1}$

${ }^{1}$ University of Newcastle, Australia

Women are exposed to fluctuations in female hormones throughout their reproductive years. For some, these fluctuations occur in endogenous hormones during the menstrual cycle, while others are exposed to exogenous hormones in contraceptives. Especially for female athletes, it is important to consider the effects of female hormones on resistance training adaptations. The limited research on acute responses to a resistance training session over the menstrual cycle has shown conflicting results with some reporting greater growth hormone response in the luteal phase, while others showed greater potential for recovery in the follicular phase. When comparing acute responses to resistance training between oral contraceptive (OC) users and women with a natural menstrual cycle, there is also no agreement in the literature. These conflicting findings may be partly explained by methodological differences between studies. For research over the menstrual cycle it is important to schedule experimental sessions to coincide with the different hormonal environments. For OC research, the many different varieties of OC need to be considered. For resistance training adaptations, in particular, the different types of progestins play an important role due to differences in androgenicity. The few studies on chronic adaptations to resistance training suggest OC users may respond better to a resistance training program than women with a natural cycle. For eumenorrheic women, however, follicular phase-based training seems to result in better responses than traditional resistance training programs. Dr Dr Janse de Jonge presented these issues, concluding that, overall, it is evident that further high-quality research is needed to provide female athletes with resistance training recommendations.

Career Reflections and Future Directions: Words, Pictures, Kids and Trees

Professor Zoe Knowles ${ }^{1}$

${ }^{1}$ Liverpool John Moores University, UK

In this session, Professor Knowles outlined her career pathway over 23 years through many 'firsts' within research, applied practice, projects and unique roles she has created. She shared candidly the origins, successes, and challenges from this period and presented use of research methodologies including Write Draw Show and Tell, and reflective and creative approaches as 'firsts.' More recent work has seen the emergence of public, place and civic engagement in Professor Knowles' portfolio and she explained why these are important for the sport and exercise sector and especially in light of the COVID-19 recovery. Professor Knowles offered questions to the audience, which hoped to challenge, provoke and disturb conventional thinking on research motivation, engagement and career pathways.

\section{Estrogen Regulation of Musculoskeletal Function}

Associate Professor Mette Hansen, $\mathrm{PhD}^{1}$

${ }^{1}$ Aarhus University, Denmark

Gender differences are observed in regard to connective tissue structure and function, which may be linked partly to a differential sex hormonal profile. The effect of female hormones on skeletal muscle connective tissue is a puzzle. Endogenous and exogenous administrated estradiol seems to influence the tendon and ligament mechanical properties differently. The latter may be related to a combination of direct and indirect hormonal effects. In this presentation, Dr Hansen added pieces to the puzzle by showing results from primarily human cross-sectional trials and randomized-controlled trials, which have aimed to elucidate effects of estrogen and oral contraceptives on connective tissue protein turnover, tissue composition and biomechanical properties, as well as injury risk. Use of oral contraceptives seem to reduce tendon and muscle connective protein synthesis rate in young women and enhance tissue stiffness. In contrast, administration of estrogen replacement therapy to elderly hysterectomized women seems to result in higher tendon collagen synthesis rates and relatively lower tendon stiffness. Furthermore, the endogenous fluctuations in estrogen during the menstrual cycle are linked to a greater knee joint laxity and higher risk of sustaining an anterior cruciate ligament injury in eumenorrheic young athletes. In conclusion, several findings indicate that endogenous and exogenous females influence the ligaments and tendon structure and biomechanical properties. This raises several questions to be answered in future research studies to be able to give sex-specific advice to the female athlete regarding participation in sport to improve health and performance.

\section{\#TimeToSeaChange: Crossing Oceans for Women in Sport}

\section{Victoria Evans ${ }^{1}$}

\section{${ }^{1}$ Red Bull Media House}

In difficult times it is easy to revert to traditional ways of doing things. Whether that is women not being active, or professional sport being a female-free zone, during the Coronavirus pandemic, huge setbacks to the momentum previously built towards developing the culture of sport have been seen. As part of this presentation, Victoria Evans explained how, having worked as a sports lawyer for the last decade, and how having experienced sport as a powerful vehicle for change in her own life, driving progress in this sector is a cause that she feels incredibly passionate about. Victoria explained how undertaking roles at Adidas, Red Bull and UEFA has enabled her to see first-hand how the culture around women's sport is starting to change, but that there is a long road ahead. She asserted that to truly optimize women's athletic success and their participation, development needs to be led from the top. This presentation also explored why continued investment in women's sport as a growth sector makes sense for all stakeholders and how every voice can have impact in driving positive change. On this last point, Victoria explained how she walks the walk, since the presentation also looked at her preparations for a challenge of extreme magnitude - solo rowing the Atlantic - in a bid to deliver a core message about women using sport and challenge to empower and create change.

\section{Getting to the Top and Staying There: Experiences of Elite Qatari Female Athletes}

Hanna Gildam-Clark ${ }^{1}$

${ }^{1}$ HGC Sports Performance 
Prior to COVID-19, sport participation rates for females in Qatar were low. Fundamental to investigating the reason for this was understanding the motives of current elite female athletes, particularly because they have spent years in the realm of Qatari sport in order to achieve elite status. The results revealed that elite Qatari female athletes' motivation towards their sports participation were multidimensional and highly self-determined. Any barriers they encountered did not affect their sustained commitment towards their sporting endeavors. Since becoming an elite athlete for the Qatar National Women's Rugby 7s Team, In this presentation, Hanna Gildam-Clark explained how she has been able to understand and witness first-hand the results of her existing study, especially during the Coronavirus pandemic. Furthermore, she explained how she underwent surgery on an existing injury prior to full lockdown. To aid her recovery, she showed how she has been utilizing her sport psychology background, previous injury experience and her research. She asserted that the tools and skills she has learnt during this period will be useful and should be considered for all female athletes on their return to play following this pandemic. Other recommendations for sporting organizations to further increase the promotion of female sport worldwide were also discussed.

\section{Opportunities and Challenges for Women in Sport Leadership in the} COVID-19 World

\section{Professor Laura J. Burton ${ }^{1}$ \\ ${ }^{1}$ University of Connecticut, USA}

In the midst of the global pandemic, gendered leadership beliefs and norms like "think manager-think male and think crisis-think female" have been amplified. This presentation addressed two significant factors impacting women working in sport leadership. First, the COVID-19 pandemic has further exposed the invisible labor of caretaking and increased workloads for women. Prior to the pandemic, women in sport have been labelled an at-risk group for their higher levels of work-family and family-work conflict. Specifically, women, compared to men, do more unpaid and unnoticed labor, and this invisible labor becomes exhausting, undervalued, and demoralizing. Second, narratives of women's success leading their nations through the pandemic may lead to essentialist thinking that women are in some innate, biological way, better at leading in crisis compared to men. We must be aware of how gender essentialist thinking can position women into precarious leadership positions. Narratives around how strong women leaders were better able to address the COVID crisis reinforce stereotypes regarding why women may be better at leading through crises. Instead of relying on stereotypes of sport leadership post-COVID, lessons learned from the crisis offer not only ways to reimagine sport as a more equitable space but also ways to shift implicit leadership models regarding strong leaders in sport away from a model that is biased against women in sport leadership.

\section{Keeping Fit and Active: Implications and Opportunities for Women} Post Lockdown

Kate Nicholson ${ }^{1}$

${ }^{1}$ Women in Sport

The Coronavirus lockdown and restrictions have been a major disruptor to people's lives and resulted in a re-setting of priorities and forging new meaning for the future. Women have been especially impacted, with gender roles amplified and limited time to prioritize self. Worryingly, declining activity levels for women have reversed a previously positive trajectory, widening the gender gap again. Existing barriers to participation for women around self-confidence and self-efficacy have been amplified for many and return to previous activities limited by both fear and lack of availability. However, the pandemic has also brought about opportunity, with physical health and wellbeing now more important than ever before, evidence of some women experiencing being active for the first time and the emergence of creative initiatives to help women prioritize their fitness. Funded by Comic Relief, Women in Sport looked to understand the impact on women across different life stages to help determine how sport and exercise, in a world that may not return 'to normal', can be successfully managed and how to learn from and better leverage the broader value of exercise post lockdown. Now is the time, not only to quickly adapt to the changes for sport and exercise, but also to respond to the opportunity to reset and reimagine how it can evolve for women. Kate Nicholson explained the need to kick-start a step change in activity levels, no longer accepting the gender gap which leaves women less active than men throughout their lives.

\section{Open Communication Abstracts}

In addition to the keynotes, there were several high-quality presentations delivered in the open communication sessions, the abstracts of which are presented below.

\section{Exercise-Related Experiences of Female Runners During Pregnancy} and Postpartum

Izzy Moore $^{1}$, Megan James ${ }^{1}$, Emma Brockwell ${ }^{2}$, Grainne Donnelly ${ }^{3,4}$, and Joanne Perkins 5

${ }^{1}$ Cardiff Metropolitan University;

${ }^{2}$ Physiomum, private practice;

${ }^{3}$ South West Acute Hospital, Western Health and Social Care Trust, N. Ireland;

${ }^{4}$ University of Ulster;

${ }^{5}$ Mumma physio, private practice

Prior to developing prenatal and postpartum interventions to support females to undertake exercise and running, it is important to understand what females currently do and what guidance is provided. The study aimed to assess the exercise-related experiences of, and guidance provided to, female runners during pregnancy and postpartum. An online survey was devised by expert pelvic health physiotherapists and human movement scientists and distributed via social media channels. Proportions (\%) were calculated and content analysis undertaken for prenatal concerns. A total of 787 postpartum females completed the survey (age $\mathrm{M}=33.7, \mathrm{SD}=$ 3.7 years). During pregnancy, only $36 \%(n=280)$ were given any prenatal exercise guidance. Of these, pelvic floor exercises were the most common guidance provided $(88 \% ; n=246)$, followed by physical activity $(55 \%$; $n=154)$ and general strengthening $(55 \% ; n=154)$. Concerns that stopped prenatal females running were: Pain/discomfort, fatigue and sickness, and fear of miscarriage/harm to the baby. The majority did not receive any postpartum guidance about returning to running $(63 \% ; n=494)$. Concerns regarding returning to running were leaking urine $(13 \% ; n=263)$, feeling heaviness in the vaginal area $(30 \% ; n=237)$ and not knowing how to return in a safe manner $(22 \% ; n=172)$. Introduction of prenatal exercise guidance as standard practice may alleviate fears that stop some females running during pregnancy. Postpartum interventions are needed to increase confidence in how to return to running safely and to address concerns regarding pelvic floor dysfunction symptoms.

\footnotetext{
The Effects of Menstrual and Contraceptive Cycle Monitoring in Elite Soccer

Guy Pitchers ${ }^{1,2}$, Kirsty Elliott-Sale ${ }^{3}$, Thomas Donelon ${ }^{1}$, and Jamie O'Driscoll ${ }^{1}$

${ }^{I}$ Canterbury Christ Church University, UK;

${ }^{2}$ Charlton Athletic Women's Football Club, UK;

${ }^{3}$ Nottingham Trent University, $U K$
} 
Monitoring menstrual cycles (MC) and hormonal contraceptive (HC) use is promoted as best practice for practitioners working with female athletes. Research has yet to determine the effects of long-term monitoring on athletes and if any changes occur within the club environment. The aims were to: (i) gain a better understanding of athlete experiences of $\mathrm{MC} / \mathrm{HC}$ monitoring; (ii) identify athlete perceptions of $\mathrm{MC} / \mathrm{HC}$ use on training and competition performance; and (iii) explore if monitoring affects a player's ability to discuss $\mathrm{MC} / \mathrm{HC}$ use within the club. Twenty soccer players completed a daily wellness survey, including questions on their current non-hormonal/hormonal status, period/withdrawal bleed and symptoms experienced. At the conclusion of the season, players completed a questionnaire assessing their experiences of the $\mathrm{MC} / \mathrm{HC}$ monitoring. Overall, $85 \%$ of players reported that monitoring made them more conscious of their individual cycle, with $90 \%$ reporting a 'very positive' or 'positive' experience. Non-hormonal contraceptive athletes perceived a $91 \%$ and $73 \%$ impact of $\mathrm{MC}$ on training and competition performance, whilst $\mathrm{HC}$ users reported 55\% and 33\% alike. Monitoring was beneficial in enabling athletes to discuss their own cycle with other players (80\%), strength and conditioning coach (100\%) and medical staff (65\%) but not soccer coaches $(30 \%)$. Monitoring of $\mathrm{MC} / \mathrm{HC}$ use was beneficial for most athletes and staff, but more work is required to improve the relationship between athlete and soccer coach. Effects upon training and competition performance occurred in both groups but are reported to be higher in non-hormonal contraceptive athletes.

Ergonomic Fit of Equipment for Female Whitewater Kayakers: Can Changing Sitting Height Increase Paddle Stroke Efficiency?

Shelley A. L. Ellis ${ }^{1}$, Mike Lauder ${ }^{2}$, Neal Smith ${ }^{2}$, and Andrew Callaway ${ }^{1}$

${ }^{1}$ Bournemouth University, $U K$;

${ }^{2}$ University of Chichester, $U K$

Whitewater kayaking has been underrepresented in the scientific literature, largely due to its recreational nature. Whitewater kayaks are manufactured on male body specifications, due to the male-dominated history of the sport. Female kayakers must therefore adapt the kayaks to meet the demands of the environment, task, and their own anthropometry, commonly achieving this through changes to sitting height. The aim of this work was to develop an accessible method to identify the optimum sitting height for female whitewater kayakers. With institutional ethical approval, a sample of experienced female whitewater kayakers $(n=7)$ was recruited. A technique efficiency observational method was developed utilizing kinematic technique measurements and boat movement measures around four of the six degrees of freedom. Kinetic measurements from the paddles were also recorded. These markers of stroke efficiency were combined, and methods of analysis were employed in pursuit of prediction of the optimum seat raise for female whitewater kayakers. The method based on percentiles proved to be the best predictor of optimum seat raise and identified seven markers of efficiency that could be used together by kayakers and coaches to identify optimum sitting height for female whitewater kayakers. These included two-dimensional kinematic measurements of pitch, velocity change, left arm reach, and stroke length left to right, alongside a timed slalom course and kinetic measurement of both left and right paddle strokes. A change in sitting height improved paddle stroke efficiency in $85.71 \%$ of the participants.

Do More Flexible Feet Explain Differences in Injury Risks and Patterns Between Female and Male Middle-Distance Runners?

Anna Kosciuk ${ }^{1,2}$, Hannah J. Moir ${ }^{1}$, and Nicola Swann ${ }^{1}$

${ }^{1}$ Kingston University London, UK;

${ }^{2}$ Profeet Sports Lab, UK
Running-related injuries are multifactorial, and their prevalence is influenced by a variety of internal and external risk factors, such as age, training volume and distance. Even when accounting for these risk factors, females have been shown to have a greater risk of running-related injuries than males, the mechanisms of which are not clearly understood. The aim of this study was to investigate sex differences in foot mobility, lower limb function and injury history in middle-distance runners. Twenty-six runners (13 males, 13 females), who ran between $30-40 \mathrm{~km}$ per week, were assessed on foot mobility, lower limb function and gait, through a variety of static, clinically recognized tests (Foot Posture Index, Windlass Mechanism, Supination Resistance and Single Leg Squat Tests) and dynamic, two-dimensional video analysis. A history of running-related injuries was reported by $73.1 \%$ of participants. Patterns of injuries differed, such that in females, hip (33.3\%), ankle (33.3\%) and foot injuries (33.3\%) were most common and in males, knee (40\%), shank (30\%) and foot injuries $(20 \%)$ were most common. Significant differences in injury prevalence were, however, only identified for the knee and hip $(p<0.05)$. Females had more pronated and flexible feet than males, with a positive relationship between injury occurrence and supination resistance in females $(p<0.05)$. Data in this study provided provisional evidence for different foot characteristics and mechanisms of running-related injuries in female and male runners, which should be considered in training.

\section{Physical Activity Behaviors of Women in the North East During the COVID-19 UK 'Stay at Home' Restrictions}

Alison Innerd ${ }^{1}$, Lynsey Bainbridge ${ }^{1}$, Natalie Connor ${ }^{2}$, Louise Morris ${ }^{2}$, Gemma Nelson ${ }^{2}$, and Emma Giles ${ }^{1}$

${ }^{1}$ Teesside University;

${ }^{2}$ Tees Valley Sport, Middlesbrough

The World Health Organization declared COVID-19 as a pandemic in March 2020. As a result, the UK Government imposed 'stay at home' restrictions. These restrictions advised everyone to stay at home except for daily exercise, essential shopping and commuting, with public gatherings exceeding two people prohibited. This survey explored the impact of stay-at-home restrictions on physical activity (PA) behavior of people in the North East of England. An online survey was completed by adults during the first phase of the restrictions (April 29, 2020 and May $13,2020)$, reporting of their PA behaviors and changes during COVID19. Out of 1,141 responses, 825 females completed the survey (aged 1672 yr; $98 \%$ white; $95 \%$ no disability). Ninety five percent of female participants reported living in a house, with access to outdoor space. Approximately half reported living with children, with $2 \%$ living alone. Preliminary analyses showed that $73 \%$ reported PA behavior to have changed during restrictions; $50 \%$ exercised more with household members than before restrictions; $28 \%$ reported increased PA, with $25 \%$ reporting a decrease; $58 \%$ reported that PA was a priority before and during the pandemic; $82 \%$ went outside to exercise most days; $80 \%$ had never used online exercise platforms at home and $29 \%$ did not use them during restrictions. Walking was the most popular activity, with social distancing cited as a barrier to PA. Early findings provide insight to support the region's recovery strategy, particularly for local authorities, public health, and the voluntary sector to design PA services for physical and mental health.

A Co-Produced Virtual Community Health Project With Mothers of Young Children From a Disadvantaged Neighborhood to Support Healthy Lifestyle Behaviors

Paula Wittels ${ }^{1}$

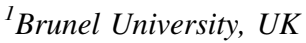


This study critically examines a co-produced virtual community health project with mothers of young children living in a disadvantaged neighborhood. Working with 20 mothers from a diverse range of ethnic backgrounds, the work involves the creation and development of a Facebook group and program of nutritional and physical activity sessions reflecting the needs and desires of the mothers. The work draws on coproduction strategies in community settings to examine the feasibility of designing, implementing and evaluating a virtual community health group for the participants. The project arose from doctoral research on the challenges faced by low socioeconomic status mothers who wish to adopt and sustain nutritional and physical activity strategies to benefit their health. Current public health interventions, particularly in the areas of sport/physical activity and diet, are commonly not relevant to mothers living in areas of disadvantage and do not take account of their views and ideas. This doctoral research identified the significance of a community participatory approach, and a supported self-help group was suggested and embraced by the mothers themselves.

Using Video Role Models to Modify Middle-Aged Women's Physical Self-Perceptions and Attitudes Towards Physical Activity Behaviors

Megan Laura Trollope ${ }^{1}$, Stuart William Flint ${ }^{2}$, Jayne Raisborough ${ }^{3}$, and Joanne Hudson ${ }^{1}$

${ }^{1}$ Swansea University, UK;

${ }^{2}$ Leeds University, $U K$;

${ }^{3}$ Leeds Beckett University, $U K$

Women are $36 \%$ more likely to be inactive than men; for middle-aged women, this is partly because of a lack of relatable role models. Middleaged women experience negative ageing stereotypes, while physical activity (PA) is promoted using "young, attractive" women. Therefore, the current study used positive role models to modify stereotypes of ageing and PA. Twenty-three middle-aged women $(\mathrm{M}=53.9, \mathrm{SD}=3.3 \mathrm{yr})$ watched a video of older women performing different life activities and were then interviewed. Three months later they attended a focus group or interview. They completed the Ageing Stereotypes and Exercise Scale and the Possible Selves scale at recruitment, pre-video and at three-month postintervention. Pre-video interviews revealed three negative PA stereotypes: 'too old to be active', 'shouldn't be in a gym' and, being a 'good mother' and three PA narrative groups: (1) Identity (identities central factor to PA participation); (2) physical (in)activity; and (3) confidence (lack of confidence underpinned physical inactivity). Three months post-intervention, Group 1 reported increased PA and more positive attitudes towards ageing, group 2 reported more positive attitudes towards PA, and Group 3 increased confidence to be physically active. Positive role models began to reduce the negative impact of ageing stereotypes on some self-perceptions and PA, thus, might offer a useful intervention. Underpinning interventions with relevant narrative themes might also help increase PA (e.g., maintaining a core identity element for a woman with an identity narrative).

Facilitating a Collaborative Learning Culture Within a Basketball Community of Practice - A Personal Account of Action Research

Sarah Wagstaff ${ }^{1}$

${ }^{1}$ Cardiff Metropolitan University, UK

Interaction with peer coaches is a useful means of informal learning and coach development and thus participating in a community of practice $(\mathrm{CoP})$ is recommended. For a lead coach, who has responsibility for mentoring coaches, there is a need for greater collaboration with coaches; the aim of the study was, therefore, to build and facilitate a $\mathrm{CoP}$. A form of emancipatory action research was used, which involved three cycles: planning, acting, observing and reflecting; each cycle included a team meeting. Personal reflective logs were kept throughout and focus groups $(n=3)$ were conducted on conclusion of the third cycle. Results provide an insight into the process of establishing a $\mathrm{CoP}$, measuring the effectiveness of the CoP and highlighting the factors which contribute to its sustainability. Key findings include 1. legitimate peripheral participation, 2 . the individual within the community and 3 . the bigger picture. Whilst a longer period was necessary to build culture, we were successful in creating a $\mathrm{CoP}$ and facilitating a collaborative learning environment. The main limitation of the actional research was the pandemic which meant we were unable to be in our practical environment. Future direction includes the need for coaches to observe each other. Furthermore, for the sustainability of the CoP, there is a need for a skilled facilitator and the CoP must continue to add value to its members and the club.

\section{A Longitudinal Case-Study Approach to Professional Female Ultra- Triathletes' Pain Management}

Britta Maria Sorensen ${ }^{1}$, Carla Meijen ${ }^{1}$, and Stacy Winter ${ }^{1}$

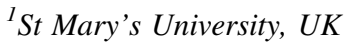

An athlete's tolerance levels to pain or discomfort can be influenced by various biopsychosocial factors. Within the burgeoning pain literature, a focus on the endurance and ultra-endurance athlete has emerged; however, the majority of this research has employed non-elite samples, has focused on male participants, and has been conducted within an experimental setting. Monitoring the pain experiences of elite female ultra-athletes over a season, and within their natural setting, would bridge this knowledge gap. This study adopted a mixed-method approach to longitudinally monitor three professional ultra-triathletes over 15-months. The aim was to adopt a biopsychosocial approach to explore their pain experiences, management, rest, recovery, and mood, and ascertain the factors that may influence them. Data collection consisted of semi-structured interviews with each athlete (six-monthly intervals) and online weekly reflective journal entries. The journal enabled athletes to write about their experiences, provide weekly training details, and reflect on pain behavior self-report questions relating to their self-efficacy, emotion, and control of pain or discomfort for that week. Three inventories were used to measure rest and recovery, mood, and pain: Recovery-Stress Questionnaire for Athletes (RESTQ-SPORT), Brunel Mood Scale (BRUMS), and the McGill Short form, completed weekly via an online link. Lastly, at sixmonthly intervals, the Sports Inventory for Pain-25 (SIP) was completed online. Due to the longitudinal nature of this study, data collection is still ongoing. A 'snapshot' focusing on how the hormonal cycle can influence mood and pain tolerance for these athletes is discussed.

\section{Gender, Leadership and Governance in English National Governing} Bodies of Sport: Formal Structures, Rules, and Processes

Lucy Piggott $^{1}$ and Jordan Matthews ${ }^{2}$

${ }^{I}$ University of Hertfordshire, UK;

${ }^{2}$ University of Chichester, $U K$

We explore the extent to which the administrative and governance hierarchies, rules, and processes of England Golf and the Lawn Tennis Association reproduce or resist gender segregation and male dominance within their leadership and governance. Drawing on Bourdieu's theory of practice, we seek to expand upon current literature in the field to better understand the workings of gender power relations at the structural level of organizational practice. Semi-structured interviews with male and female leaders were supplemented by an analysis of formal documents from the two organizations. We found that gender power relations that privilege dominant men were simultaneously conserved and resisted within the two National Governing Bodies (NGBs) in the paid and voluntary workforce. 
Vertical gender segregation, the exclusion of women from the highest leadership positions, and the gendered distribution of tasks were impacted by the NGBs' administrative and governance structures, rules, and processes. Whilst resistance to male-dominated leadership and governance was evident, transformational organizational change was found to be lacking. This highlighted the limitations of leadership and governance changes being primarily driven through top-down, policy-based approaches. We emphasize the importance of a combined approach at the structural, cultural, and individual levels to enable sustainable and transformational organizational change.

\section{"There Has Been No Difference Between Male and Female Athletes in the VBL [German Volleyball League] for Almost 15 Years" - Between Equal Visibility and Objectified Staging of Female Athletes in Profes- sional Volleyball Teams in Germany}

Christina Plath ${ }^{1}$

${ }^{1}$ University of Vechta, Germany

While sports reporting is often characterized by binary gender stereotypes, the marginalization of non-male performance and a so called "male gaze", this does not seem to apply to the professional volleyball sport in Germany at first glance. At national and international level, the matches and tournaments are represented at least equally or with a 1:4 ratio in favor of the women's teams in the media. Also, the female athletes are represented in active poses. In line with this notion, the German Volleyball League (VBL) and the German Volleyball Federation (DVV) communicate that they have been realizing gender equality for 15 years. On the basis of the theory of objectification and the concept of hegemonic masculinity, the staging of the volleyball sport by the association and clubs is examined in terms of content analysis, in addition to media visibility. While more than half of the volleyball club members are female athletes, women are strongly marginalized in all leadership/responsibility positions. Furthermore, there is a strong contrast between the communicated realization of gender equality from the VBL and DVV and a sexualized marketing of female athletes by their sports clubs. The comparatively high visibility of women's teams thus contributes, at least in part, to the reproduction of gender stereotypes through an objectified staging of the female athletes.

\section{COVID-19 and Elite Women's Sport: Experiences of Lockdown and} Concerns for the Future

Ali Bowes ${ }^{1}$, Jessica Piasecki ${ }^{1}$, and Lucy Lomax ${ }^{2}$

${ }^{1}$ Nottingham Trent University, UK;

${ }^{2}$ Independent Researcher/Women's Rugby Hub

The COVID-19 pandemic decimated elite level sport across the globe, although there were concerns that this impact affected women's sport much more so than for men's sport. This research considers both the impact of the enforced global lockdown on elite female athletes, examining the immediate effect on their training practices, welfare and financial situation, and their perceptions on the long-term impact on women's sport. A qualitative, online survey was used to collect anonymous data from 95 elite sportswomen. Data were coded to enable frequency analysis, and then analyzed thematically. Many sportswomen noted the positive support received from coaching networks regarding adapted, individualized training programs and their mental well-being. A reduction in time spent training, and changes to types of training, was reported. However, many felt they had less access to equipment as women, and that men's sport was a priority. Professional and semi-professional sportswomen, already operating in financially precarious circumstances, were also impacted through a reduction in match fees and/or sponsorship, or furlough. The sportswomen emphasized their concern that these two issues combined would impact women's sport more than men's sport, potentially reversing the progress made in recent years. The findings provide empirical evidence of a gendered dimension to the experiences of elite athletes during lockdown, and a concern for the future of women's sport. When elite sport re-builds post-COVID, there should be a greater awareness of the difficulties that were faced by sportswomen, and targeted action to tackle gender inequality in sport, especially around media coverage and funding/sponsorship.

\section{Gendered Curricula and Female Students' Experiences on University Sport Courses}

Philippa Velija ${ }^{1}$ and Catherine Phipps ${ }^{1}$

\section{${ }^{1}$ Solent University, UK}

Our research is guided by the following research aims: a) to understand how gender is constructed in curriculum design and b) to explore how gender is taught to undergraduate students and how they are taught to challenge existing gender relations in sport. This research is significant, as across higher education institutes in the UK more male students study on sport-related degree courses than female students, and positions in elite sport remain male dominated. In 2017/2018, 12,705 female students studied a sport-related course compared to 26,885 males. The reason for the gender gap across sport courses may be complex and multifaceted; however, in order to challenge existing ideas about female athletes and women in sport, the ways in which courses are marketed, how curriculum is designed and how students are taught about gender are all significant. This is particularly the case where students study subjects where the male body and research on male athletes are normalized, and where stereotypes about female athletes and women in sport go unchallenged. Data derived from a case-study exploring sport courses at one university are presented. This includes an analysis of curricula content, marketing of sport courses, and workshops with students on their experiences of the curricula, gender and learning spaces, to explore how issues of gender are experienced by students on sport courses.

\section{Developing a Strategy for Female Role Models in Physical Activity and Sports Promotion}

Eimear Kelly ${ }^{1}$, Aoife Lane ${ }^{1}$, Kieran Dowd ${ }^{1}$, and Katie Liston ${ }^{1}$

${ }^{1}$ Athlone Institute of Technology, Ireland

The aim of this study is to create a role model sports-based intervention to promote physical activity (PA) and sport participation in female youth. A systematic literature review will be conducted on role modelled interventions, as well as a national survey to identify the nature and experience of role models in Irish society. In Ireland, 9\% of girls (aged $10-18$ years) meet the daily recommendations for PA and $23 \%$ participate in sports at least once per week. Among adult females, $26 \%$ meet PA recommendations, and in 2017, 69.9\% of 16-19-year olds participated in sport, which decreased with age to $35.9 \%$ among those $\geq 35$ years. Just $3 \%$ of sports print media is devoted to women's sport; $23 \%$ of those in leadership, through Chief Executive Officer positions and National Governing Body board membership, are female, and the ratio of male to female coaches in sport is 2.5:1. Role models are star athletes, loyal players, coaches, and parents who inspire and empower others through their own participation and involvement in sport. It has been shown that girls are less likely than boys to have sporting role models and that children with role models are more physically active. There is a lack of evidence for how role model-led interventions can promote PA, but evidence suggests that role models must be relevant and accessible. In Ireland, Gaelic Games are the most popular team sports for girls. The local, amateur nature of these games provides an ideal setting for a successful trickle-down effect on sport/PA. 
Understanding Perception and Factors Influencing Levels of Physical Exercise in Both Male and Female Patients With Psoriatic Arthritis: A Qualitative Study

Millie Meadows ${ }^{1}$, Jeremy Moody ${ }^{1}$, Diane Crone ${ }^{1}$, Joseph Esformes ${ }^{1}$, and Andy Kelly ${ }^{1}$

${ }^{1}$ Cardiff Metropolitan University, UK

Psoriatic arthritis (PsA) is a distinct variant of inflammatory arthritis of unknown etiology, associated with the skin disease, psoriasis. Physical exercise is known to improve physical function and reduce fatigue and disease-related activity in patients with rheumatic conditions, including PsA. Nevertheless, the majority of patients with PsA are physically inactive, indicating possible barriers when it comes to physical exercise. Pain, fatigue and time have been identified as barriers to physical exercise in PsA patients. Further exploration of these findings is needed to further elicit patients' preferences and opinions in order to increase patients' participation and adherence to physical exercise, thus reducing fatigue and disease-related activity. This qualitative study looks to assess barriers and facilitators, explore patients' preferences and opinions relating to physical exercise prescription/content, and analyze the potential differences between male and female perceptions. This study will be useful in developing interventions tailored to the patient's needs, and thus more likely to be successful when it comes to exercise prescription and adherence. Demographic variables, psychological status and disease characteristics will be analyzed. The results from this study will further inform an exercise intervention involving the comparison of different training modalities and an analysis of the physiological and psychological effects, thus informing clinical practice in terms of appropriate and individualized exercise prescription to PsA patients.

How Is the Elite Cycling World Changing for the French Female Cyclist? A Cultural and Comparative Snapshot of Progress So Far

Britta Maria Sorensen ${ }^{1}$

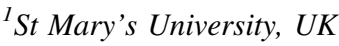

There are many sociocultural and historical factors that influence the inequalities for the elite female cyclist in France. A new association is working hard to address these inequalities and provide them with a professional status similar to that of the male cyclists. The aim of this presentation is to explore and update the latest and ongoing work of this association, to discuss some female-specific issues from the cyclist's perspective, e.g., needing education on nutrition and training or dealing with harassment. This talk offers insight and cultural comparison for female cyclists, for the same objective of smashing the inequalities in sport. For example, the association's aim is to professionalize women's cycling in France and improve cycling conditions for women around the world with the women's CPA (Association of Pro Riders): The international association CPA Women defends the interests of female cyclists, is committed to improving their working conditions and represents their members in cycling institutions. In 2017, CPA opened its doors to a number of high-level female athletes striving to find an association to represent them and their interests, much in the same way as male athletes are represented. This is how CPA Women came to life as the women's chapter of the International Riders' Association, which is open to female athletes only. The French association-AFCC finalized the protocols that helped prepare, via a working group, so that athletes can return to road racing safely - not an easy task, since races are all over the world.

Enabling Resistance Training in Young Female Soccer Players: An Examination of Coaching Attitudes

Christo Manginis ${ }^{1}$ and Alex Blackett ${ }^{1}$

${ }^{1}$ Staffordshire University, $U K$

This study sought to uncover why young female soccer players rarely resistance train despite its unique benefits; in addition, we wished to investigate what might enable players to undertake resistance training. Thirteen club and/or high school varsity coaches of female soccer players were asked, via semi structured interviews, about their perceptions and potential implementations of resistance training. Thematic analysis was used to analyze the interviews revealing many positive opinions about resistance training. Experience and personal research led to knowledge and understanding of the physical and mental benefits of resistance training, which enabled coaches to promote and engage this form of training. Coaches were also empowered by creativity and close relationships with resistance training experts. Injury and social fears, such as the loss of femininity, were major barriers for coaches and players. Lack of resources also made it difficult for coaches to promote resistance training and for girls to engage. Lastly, coaches were hesitant to encourage young athletes, of about 13 years and below, to engage in resistance training because it was deemed either especially dangerous for these athletes and/or unnecessary. This notion is at odds with the research and may cause children to either start resistance training late or to never resistance train at all. This research can help coaches to overcome their biases regarding resistance training and improve their practices. 